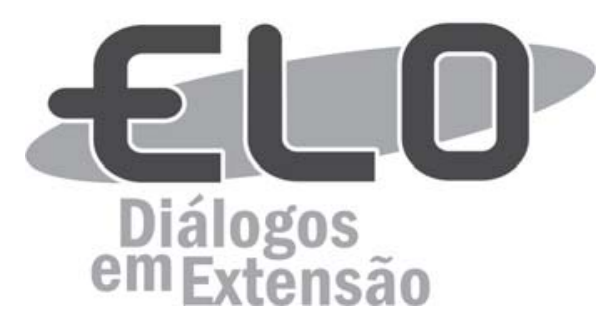

\title{
Relato de experiência do projeto de extensão Primeiros Socorros: educando a comunidade e os profissionais de saúde do municipio de Viçosa-MG e região
}

\author{
Flávia Batista Barbosa de Sá Diaz¹, Sarah de Moraes Alves², Aline Aparecida de Souza Godinho", \\ Gabriella Machado de Sousa Oliveira ${ }^{4}$, Marina Talma Torres Santos Ferreira ${ }^{5}$, Nicoli Souza Carneiro ${ }^{6}$
}

\begin{abstract}
Resumo: É através da educação em saúde que o enfermeiro exerce seu papel de educador capacitando a sociedade para atuar de forma qualificada no atendimento dos primeiros socorros às vítimas em situações de urgência e emergência. O objetivo deste trabalho é descrever as atividades desenvolvidas pelos integrantes do projeto de extensão "Primeiros Socorros: educando a comunidade e os profissionais de saúde do município de Viçosa-MG e região". Trata-se de um relato de experiência das vivências dos integrantes do referido projeto no período de março a outubro de 2018. Foram realizadas quatro reuniões organizacionais, duas oficinas educativas e dez capacitações teórico-práticas, totalizando 226 participantes de públicos diversos. Esta vivência proporcionou uma aproximação da comunidade acadêmica com a sociedade de Viçosa e região, socializando o conhecimento científico adquirido na universidade, buscando melhores atendimentos em Primeiros Socorros.
\end{abstract}

Palavras-chave: Enfermagem em Emergência. Educação em Saúde. Reanimação Cardiopulmonar.

Área Temática: Saúde.

\section{Extension's project case report: First Aid: teaching Viçosa's-MG city health professionals, community and region}

\begin{abstract}
Health education is the way nurses act as an educator, by capacitating the society to act effectively on first aid assistance to urgent and emergent situations victims. The goal of this research is to report the realized activities by the members of the extension's project: "First Aid: teaching Viçosa's-MG city health professionals, community and region". It is a case report about the members' lived experiences of the extension project during the period of March to October 2018. To do this research we take four organizational reunions, two educative workshops and 10 theoretical-practical capacitation, in which 226 participants from several groups learned. The experience provided an approximation of the academic community with the society of Viçosa and region, socializing the scientific knowledge acquired at the university, and seeking better care in First Aid.
\end{abstract}

Keywords: Emergency Nursing. Health Education. Cardiopulmonary Resuscitation.

Thematic area: Agroecology.Relacionessolidarias, producciónsostenible y consumoconsciente: experiencia del Quintal Solidario - Feria de EconomiaSolidaria y Agricultura Familiar

Resumen: Es a través de la educación sanitaria que las enfermeras desempeñan su papel de educadores, lo que permite a la sociedad actuar de manera calificada para brindar primeros auxilios a las víctimas en situaciones de urgencia y emergencia. El objetivo de este documento es describir las actividades desarrolladas por los

Docente do Departamento de Medicina e Enfermagem da Universidade Federal de Viçosa - UFV, Viçosa - MG, Brasil.

${ }^{2}$ Docente do Departamento de Medicina e Enfermagem da Universidade Federal de Viçosa - UFV, Viçosa - MG, Brasil. Endereço: Departamento de Medicina e

Enfermagem, Avenida Peter Henry Rolfs, s/n. Campus Universitário - Viçosa, MG. CEP: 36570-900, Telefone: (31) 36125507. E-mail: enfsarah@gmail.com

${ }^{3}$ cadêmica de Enfermagem pela Universidade Federal de Viçosa - UFV, Viçosa - MG, Brasil.

${ }^{4}$ Acadêmica de Enfermagem pela Universidade Federal de Viçosa - UFV, Viçosa - MG, Brasil.

${ }^{5}$ Acadêmica de Enfermagem pela Universidade Federal de Viçosa - UFV, Viçosa - MG, Brasil.

${ }^{6}$ Enfermeira. Residente em Atenção Primária e Saúde Coletiva pela Universidade de São Paulo - USP. 
miembros del proyecto de extensión "Primeros auxilios: educación de la comunidad y profesionales de la salud de Viçosa-MG y la región". Este es un informe de experiencia de las experiencias de maestros y estudiantes de este proyecto de extensión de marzo a octubre de 2018. Hubo 04 reuniones de organización, dos talleres educativos y diez capacitación teórico-práctica, con un total de 226 participantes del público misceláneo. Esta experiencia proporcionó una aproximación de la comunidad académica con la sociedad de Viçosa y la región, socializando el conocimiento científico adquirido en la universidad, buscando una mejor atención en primeros auxilios.

Palabras clave: Primeros Auxilios. Enfermería de Urgencia. Educación en Salud.

\section{Introdução}

Os Primeiros Socorros (PS) são definidos como atendimento inicial realizado até a chegada de atendimento avançado às vítimas em situação de emergência, como: hemorragia, convulsões, queimaduras, mal súbito, acidentes, parada cardiorrespiratória, asfixia, entre outras. Considerando que estes tipos de ocorrências colocam a vida de indivíduos em risco, podendo ocorrer a qualquer momento, em qualquer lugar, decorrentes de diversas causas, acometendo diversas faixas etárias e classes sociais, é imprescindível que um socorrista capacitado preste atendimento mais qualificado a estas vítimas. Assim sendo, intervenções básicas em situações de emergência, quando realizadas de imediato, minimizam o sofrimento, previnem sequelas e complicações futuras e podem salvar vidas (FERREIRA et al., 2017; SILVA et al., 2018).

O conhecimento a respeito dos PS oferece um suporte adequado para agir em situações críticas. Dessa forma, prestar socorro não se pauta unicamente na realização dos procedimentos em PS, mas também na avaliação do estado geral da vítima, do local onde se encontra e do momento adequado para solicitar ajuda (RAGADALI FILHO et al., 2015).

É fundamental que esse tipo de conhecimento seja disseminado para toda sociedade, no intuito de tornar as pessoas capacitadas a prestar os PS em situações de urgência e emergência, evitando complicações desnecessárias, decorrentes de ações inadequadas e despreparadas (GALINDO NETO et al, 2017; RAGADALI FILHO et al., 2015).

Esta realidade torna clara a necessidade de formação de profissionais de saúde aptos a intervirem em situações emergenciais e a capacitarem os diversos públicos para atuarem de forma ágil, adequada e pautada em uma prática baseada em evidências científicas na realização de ações em PS. Mediante ao referido cenário, o enfermeiro ocupa uma posição ímpar enquanto educador em saúde, sendo essa uma de suas habilidades presentes no escopo de atuação profissional, a qual permite a melhora na qualidade de vida da população por meio do compartilhamento de saberes (OLIVEIRA et al, 2015; PEREIRA et al, 2015).

\section{Objetivo}

Descrever as atividades desenvolvidas pelo projeto de extensão "Primeiros Socorros: educando a comunidade e os profissionais de saúde do município de Viçosa-MG e região", que tem como objetivo capacitar a comunidade e os profissionais de saúde do município na atuação em situações de urgência e emergência da sociedade nos processos de tomada de decisão.

\section{Metodologia}

Trata-se de um relato de experiência das vivências de duas docentes do curso de Enfermagem da Universidade Federal de Viçosa (UFV), coordenadoras do projeto de extensão universitária "Primeiros Socorros: educando a comunidade e os profissionais de saúde do município de Viçosa-MG e região", 14 discentes do curso de Enfermagem da UFV e uma bolsista do Programa Institucional de Bolsas de Iniciação à Extensão Universitária (PIBEX) no período de março a outubro de 2018.

O projeto realizou quatro reuniões para fins organizacionais, duas oficinas educativas com os membros do projeto e dez capacitações com o total de 193 participantes, que incluíram crianças, adultos de diversas áreas e profissionais de saúde. Além disso, o projeto também participou do evento público "Ciência na Praça", realizado no município de Viçosa-MG, com a presença de 33 pessoas, totalizando um quantitativo de 226 pessoas capacitadas no período de oito meses. 
As atividades do projeto abrangeram: reuniões periódicas presenciais para apurar a demanda de capacitações, sua organização logística, bem como as estratégias metodológicas a serem utilizadas; capacitações educativas dos membros do projeto; confecção de materiais a serem utilizados durante as capacitações; levantamento de recursos financeiros; capacitações em PS para a comunidade local; e apresentação de trabalhos científicos.

As capacitações para a comunidade foram teórico-práticas. A parte teórica contou com apresentação de slides confeccionados pelos membros do projeto, a qual foi desenvolvida e revisada frequentemente, utilizando-se referências científicas atualizadas. A parte prática contou com atividades lúdicas, simulações realísticas e treinamento de técnicas, estimulando-se sempre a participação ativa dos educandos. Para isso, utilizou-se os seguintes materiais: ataduras, talas, bonecos tipo bebê, manequins para ressuscitação cardiopulmonar, simulador do desfibrilador externo automático e animais peçonhentos (cobras, aranhas, escorpiões e outros artrópodes), que foram fornecidos e supervisionados por estudantes de graduação em Biologia, membros voluntários do Museu de Zoologia da UFV.

Os temas trabalhados nas capacitações foram: síncope, crise convulsiva, hemorragia e epistaxe, queimaduras (calor e frio), choque, intoxicações exógenas, fraturas, luxações, entorses, acidentes com animais peçonhentos, engasgo, parada cardiorrespiratória (Suporte Básico e Avançado de Vida) e atendimento pré-hospitalar ao politraumatizado. As capacitações foram direcionadas de acordo com a necessidade do público, que foi composto por adultos, crianças, leigos e profissionais de saúde.

No início e no final das capacitações, foi aplicado um questionário criado por membros do projeto para avaliar os conhecimentos prévios e adquiridos após a capacitação (pré e pós-teste), conforme mostrado no Quadro 1. O questionário utilizado não foi identificado, estando em consonância com o Art. $1^{\circ}$ - parágrafo único, item VII da Resolução 510/2016 do CONEP, o qual suspende a necessidade de submissão do projeto ao Comitê de Ética e Pesquisa com Seres Humanos em situações de "atividade realizada com o intuito exclusivamente de educação, ensino ou treinamento sem finalidade de pesquisa científica, de alunos de graduação, de curso técnico, ou de profissionais em especialização" CEP/ CONEP (BRASIL, 2016).

\section{Quadro 1 - Questionário de Pré e Pós-Teste Aplicado nas Capacitações}

Escreva abaixo SIM (SE VOCÊ SABE), NÃO (SE NÃO SABE) e PARCIAL (SE SABE MAIS OU MENOS):

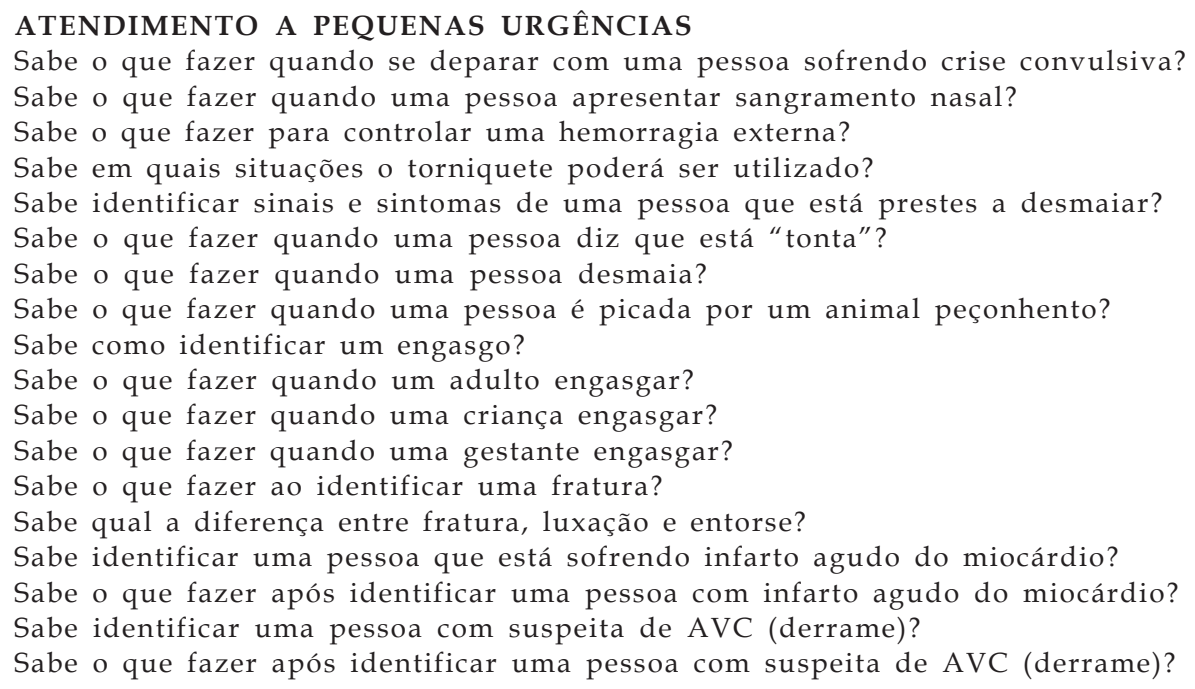

\section{AVALIAÇÃO DA ATIVIDADE}

O conteúdo exposto foi claro e objetivo?

A forma da exibição foi clara e compreensiva?

O tempo de duração da atividade foi eficaz?

O treinamento prático foi proveitoso?

A temática foi útil para a minha prática hospitalar?

Os palestrantes demonstraram conhecimento técnico e científico?

Todas as dúvidas foram sanadas durante a capacitação?

Por que você se inscreveu no curso de Primeiros Socorros?

Sua expectativa foi atendida com o curso?

Sugestões: 


\section{Resultados e Discussão}

Considerando os dados coletados no pré e pós teste, observou-se que a maioria dos participantes não possuía conhecimento prévio adequado sobre PS, inclusive entre os profissionais de saúde. Discentes do projeto perceberam, através do relato informal dos participantes após a capacitação, que houve maior conhecimento teórico e prático, além de maior segurança para intervir em situações de urgência e emergência por parte dos educandos.

Reforçando este relato, em estudo, Alves (2016) mostra que participantes de capacitações sobre Suporte Básico de Vida expressaram que o curso trouxe maior segurança, confiança, desejo por aprender, aptidão, menos dúvidas e mais tranquilidade; e também relataram que se sentem mais preparados para atuar em atendimento a vítimas em urgência.

Segundo Ferreira et al. (2017), a capacitação de público leigo (pessoas que não são profissionais de saúde) é de suma importância, visto que qualquer pessoa está sujeita a situações emergenciais em diferentes locais e, sabendo agir de forma correta, pode-se obter um resultado satisfatório em benefício da vítima.

Se não houver uma intervenção rápida e correta, uma parada cardíaca súbita pode resultar em morte em pouco tempo. É importante que se inicie a cadeia de sobrevivência rapidamente, que inclui: diagnóstico precoce da parada cardíaca, acesso rápido ao sistema de resposta a emergências, Ressuscitação Cardiopulmonar (RCP) imediata e de alta qualidade e rápida desfibrilação, se necessário (ONAN; SIMSEK, 2017).

O tempo entre a parada cardíaca e o início da RCP é crucial para otimizar os resultados do paciente. As taxas de sobrevivência aumentam de duas a três vezes quando se inicia a RCP imediatamente (BOET et al., 2017).

O enfermeiro deve desenvolver a habilidade de promover ações de Educação em Saúde como fator indispensável em sua prática profissional. Quando estudantes, professores e funcionários estão capacitados a agir em situações emergenciais, tem-se maiores chances de promover um ambiente mais seguro, tanto no que diz respeito à prevenção de acidentes, como nas ações a serem realizadas mediante situações críticas já constituídas (GALINDO NETO et al, 2017).

Investir na capacitação dos estudantes de Enfermagem, tendo-os como agentes de mudança, é investir nos mesmos com diversos papéis, inclusive de também serem educadores (NACCARELLA; BUTTERWORTH; MOORE, 2016). Freire (2002) afirma que ensinar e aprender não podem existir separadamente, uma vez que tais ações estão intrinsecamente interligadas e são mutuamente dependentes. O aprendizado é um processo que não se limita em si mesmo, abrangendo dimensões não só de uma organização linearizada, mas sim longitudinal e transversal, perpassando alunos e professores e alternando dinamicamente esses papéis sociais (FREIRE, 2002).

Observou-se, assim, a importância deste projeto também como forma de conscientizar e capacitar futuros profissionais enfermeiros a trabalharem educação em saúde, visando melhor assistência à saúde da população.

\section{Conclusões}

Os membros do projeto vivenciaram as particularidades da educação em saúde tendo contato com a comunidade, desenvolvendo suas habilidades em PS e propagando informações de interesse público. A análise dos pré e pós testes dos participantes demonstrou um resultado positivo, refletindo em maior compreensão dos mesmos para atuarem frente às situações emergenciais. Desta forma, esta vivência proporcionou uma aproximação da comunidade acadêmica com a sociedade de Viçosa e região, socializando o conhecimento científico adquirido na universidade e buscando melhores atendimentos em Primeiros Socorros.

\section{Referências}

ALVES, S. M. Teoria da Aprendizagem Transformativa aplicada em capacitação sobre Suporte Básico de Vida no contexto de trabalho de uma indústria metalúrgica. 2016. 134 f. Dissertação (Mestrado) Escola de Enfermagem de Ribeirão Preto da Universidade de São Paulo (EERP-USP), Ribeirão Preto, 2016. 
BOET, S. et al. Retention of Basic Life Support in Laypeople: Mastery Learning vs. Time-based Education. Prehospital Emergency Care, v. 21, n.3, p.362-377, 2017. Disponível em: https:// www.ncbi.nlm.nih.gov/pubmed/28059603. Acesso em: 15 out. 2018.

BRASIL, Conselho Nacional de Saúde, Ministério da Saúde, Resolução No 510, de 07 de abril de 2016.

FERREIRA, M. G. N. et al. O leigo em primeiros socorros, uma revisão integrativa. Revista de Ciências de Saúde Nova Esperança, Paraíba, v. 15, n. 3, p. 12-20, dez. 2017. Disponível em: http:// www.facene.com.br/wp-content/uploads/2010/11/Artigo-02.pdf. Acesso em: 15 out. 2018.

FREIRE, P. Pedagogia da autonomia: saberes necessários à prática educativa. 25 ed. São Paulo SP: Paz e terra. 2002.

GALINDO NETO, N. M. et al. Primeiros socorros na escola: construção e validação de cartilha educativa para professores. Acta Paulista de Enfermagem, Pernambuco, v. 30, n. 1, p. 87-93, mar, 2017. Disponível em: http://www.scielo.br/pdf/ape/v30n1/1982-0194-ape-30-01-0087.pdf. Acesso em: 15 out. 2018.

NACCARELLA, L.; BUTTERWORTH, I.; MOORE, T. Transforming health professionals into population health change agents. Journal of Public Health Research, v.5, n.643, p.21-26, 2016. Disponível em: https://www.ncbi.nlm.nih.gov/pubmed/27190973. Acesso em: 15 out. 2018.

OLIVEIRA, M. R. et al. Concepção de graduandos de enfermagem sobre a prática de educação em saúde em primeiros socorros. Revista da Rede de Enfermagem do Nordeste, Fortaleza, v. 16, n. 2, p. 150-158, mar/abr. 2015. Disponível em: <http://repositorio.ufc.br/bitstream/riufc/12643/1/ 2015_art_mroliveira.pdf>. Acesso em 28 out. 2018.

ONAN, A.; SIMSEK, N. Interprofessional education and social interaction: The use of automated external defibrillators in team-based basic life support. Health Informatics Journal, v.25, n.1, p.139$148,2017$.

PEREIRA, K. C. et al. A construção de conhecimentos sobre prevenção de acidentes e primeiros socorros junto ao público leigo. Revista de Enfermagem do Centro-Oeste Mineiro, Divinópolis, v. 5, n. 1, p. 1478-1485, mar/abr. 2015. Disponível em: http://www.seer.ufsj.edu.br/index.php/recom/article/ view/456/837. Acesso em: 15 out. 2018.

RAGADALI FILHO, A. et al. A importância do treinamento de primeiros socorros no trabalho. Revista Saberes, São Paulo, v. 3, n. 2, p. 114-125, jul/dez. 2015. Disponível em: https:/ / facsaopaulo.edu.br/ wp-content/uploads/sites/16/2018/05/ed3/10.pd>. Acesso em 15 out. 2018.

SILVA, D. P. et al. Primeiros socorros: objeto de educação em saúde para professores. Revista Enfermagem UFPE OnLine, Recife, v. 12, n. 5, p.1444-1453, mai. 2018. Disponível em: https:// periodicos.ufpe.br/revistas/revistaenfermagem/article/viewFile/234592/28912. Acesso em: 15 out. 2018.

Recebido para publicação em 30/10/2018 e aprovado em 14/05/2019. 